\title{
Decomposição de Matrizes Antiautossimilares em Matrizes de Posto-1 e Aplicações em Processamento de Sinais
}

\author{
H. B. A. Barbosa, L. B. da Silva, e R. M. Campello de Souza .
}

Resumo-Este trabalho define uma nova espécie de simetria em matrizes, através do conceito de matrizes antiautossimilares. Além disso, é proposta uma forma sistemática de expressar uma matriz antiautossimilar como combinação linear de matrizes de posto-1. Através da decomposição obtida, algoritmos rápidos para a multiplicação de quatérnios e octônios são mostrados, ilustrando o método proposto.

Palavras-Chave-Matrizes antiautossimilares, algoritmos rápidos, decomposição de matrizes, matrizes de posto-1, complexidade multiplicativa, números hipercomplexos.

Abstract-This work defines a new kind of matrix symmetry, through the concept of anti-self-similar matrices. A method of expanding an arbitrary anti-self-similar matrix as a linear combination of rank-one matrices is presented. As an illustration of the proposed techniques, fast algorithms for computing quaternion and octonion multiplications are shown.

Keywords-Anti-self-Similar matrices, fast algorithms, rankone matrices, multiplicative complexity, hypercomplex numbers.

\section{INTRODUÇÃO}

Algoritmos rápidos são fundamentais para implementação eficiente de ferramentas importantes em engenharia, tais como transformadas discretas e filtros digitais. Classes de algoritmos rápidos muito utilizados como as FFTs (Fast Fourier Transforms) de base 2 buscam explorar simetrias matriciais e utilizam a estratégia de dividir para conquistar, que consiste em dividir problemas maiores em vários subproblemas menores do mesmo tipo, resolver os problemas simplificados e reunir as soluções obtidas. Nesse contexto, é interessante identificar e propor novas formas de simetria em matrizes para obtenção de algoritmos de baixa complexidade.

Lafon [1] mostrou que a computação de baixa complexidade multiplicativa de formas bilineares está intimamente ligada ao posto tensorial das matrizes associadas a essas formas e pode ser implementada por meio da decomposição dessas matrizes em matrizes de posto-1. Em [2], foi mostrado como se obter um algoritmo rápido para qualquer transformada discreta linear a partir da decomposição formada pelo menor número de matrizes de posto-1 para um dado conjunto finito de matrizes racionais.

H. B. A. Barbosa, e-mail: helder.alves@ufpe.br; L. B. da Silva, Departamento de Eletrônica e Sistemas, Universidade Federal de Pernambuco / UNIFBV - Centro Universitário Boa Viagem, Recife - PE, e-mail: luciano.lucianobarboza@gmail.com; R. M. Campello de Souza, e-mail: ricardo.csouza@ufpe.br, Departamento de Eletrônica e Sistemas, Universidade Federal de Pernambuco, Recife - PE.
Neste artigo, é apresentada uma nova espécie de simetria matricial, através do conceito de matrizes antiautossimilares. Além disso, através da estratégia de dividir para conquistar, é obtido um procedimento sistemático para a decomposição de matrizes antiautossimilares em matrizes de posto-1. A Seção II conceitua matrizes antiautossimilares, enquanto a Seção III mostra como decompor uma matriz antiautossimilar em matrizes de posto-1. Na Seção IV, são apresentados cenários possíveis de aplicação e são obtidos algoritmos rápidos utilizando a metodologia exposta. Na Seção V são apresentadas as conclusões do artigo.

\section{Matrizes Antiautossimilares}

Definição 1: Uma matriz A de ordem 2 é antiautossimilar se apresenta a seguinte forma:

$$
\left[\begin{array}{cc}
z_{1} & z_{2} \\
-z_{2} & -z_{1}
\end{array}\right]
$$

Em geral uma matriz de ordem $2^{n}$ é denominada antiautossimilar se tem a forma

$$
\left[\begin{array}{cc}
\mathbf{A}_{11} & \mathbf{A}_{12} \\
-\mathbf{A}_{12} & -\mathbf{A}_{11}
\end{array}\right]
$$

em que $\mathbf{A}_{11}$ e $\mathbf{A}_{12}$ são antiautossimilares de ordem $2^{n-1}$. Uma matriz antiautossimilar de ordem $2^{n}$ é denominada aqui por $A A_{2^{n}}$.

Exemplo 1: A matriz $\mathbf{M}_{4}$

$$
\left[\begin{array}{cc:cc}
-b_{1} & -b_{2} & b_{3} & b_{4} \\
b_{2} & b_{1} & -b_{4} & -b_{3} \\
-- & -- & -- & -- \\
-b_{3} & -b_{4} & b_{1} & b_{2} \\
b_{4} & b_{3} & -b_{2} & -b_{1}
\end{array}\right]
$$

é uma matriz $A A_{4}$.

Exemplo 2: A matriz $\mathbf{M}_{8}$

$\left[\begin{array}{cc|cc|cc|cc}-b_{1} & -b_{2} & b_{3} & b_{4} & b_{5} & b_{6} & b_{7} & b_{8} \\ b_{2} & b_{1} & -b_{4} & -b_{3} & -b_{6} & -b_{5} & -b_{8} & -b_{7} \\ -- & -- & -- & -- & -- & -- & -- & -- \\ -b_{3} & -b_{4} & b_{1} & b_{2} & -b_{7} & -b_{8} & -b_{5} & -b_{6} \\ b_{4} & b_{3} & -b_{2} & -b_{1} & b_{8} & b_{7} & b_{6} & b_{5} \\ -- & -- & -- & -- & -- & -- & -- & -- \\ -b_{5} & -b_{6} & -b_{7} & -b_{8} & b_{1} & b_{2} & -b_{3} & -b_{4} \\ b_{6} & b_{5} & b_{8} & b_{7} & -b_{2} & -b_{1} & b_{4} & b_{3} \\ -- & -- & -- & -- & -- & -- & -- & -- \\ b_{7} & b_{8} & b_{5} & b_{6} & b_{3} & b_{4} & -b_{1} & -b_{2} \\ -b_{8} & -b_{7} & -b_{6} & -b_{5} & -b_{4} & -b_{3} & b_{2} & b_{1}\end{array}\right]$.

é uma matriz $A A_{8}$. 


\section{RESUltados IMPORTANTES SOBRE AS MATRIZES ANTIAUTOSSIMILARES}

Teorema 1: Seja A uma matriz $A A_{2^{n}}$. Então existe um conjunto $\mathcal{B}_{2^{n}}=\left\{\mathbf{B}_{1}, \mathbf{B}_{2}, \ldots, \mathbf{B}_{2^{n}}\right\}$ em que cada $\mathbf{B}_{j}$ é uma matriz de posto unitário, $j=1,2, \ldots, 2^{n}$, de modo que

$$
\mathbf{A}=\sum_{j=1}^{2^{n}} \beta_{j}(\mathbf{A}) \mathbf{B}_{j}
$$

em que $\mathbf{B}_{j}=\mathbf{b}_{j}^{\prime} \otimes \mathbf{b}_{j}^{\prime \prime T}, \mathbf{b}_{j}^{\prime}=\left[b_{i}^{\prime}\right]_{2^{n} \times 1}, \mathbf{b}_{j}^{\prime \prime}=$ $\left[b_{i}^{\prime \prime}\right]_{2^{n} \times 1}, \quad b_{i}^{\prime} \in\{-1,+1\}, \quad b_{i}^{\prime \prime} \in\{-1,+1\} \quad \beta_{j}(\mathbf{A})=$ $\frac{1}{2^{n}}\left[\begin{array}{llll}z_{1} & z_{2} & \ldots & z_{2^{n}}\end{array}\right] \cdot \mathbf{b}_{j}^{\prime \prime},\left[\begin{array}{llll}z_{1} & z_{2} & \ldots & z_{2^{n}}\end{array}\right]$ é a primeira linha da matriz $\mathbf{A}$, · indica o produto interno e $\otimes$ indica o produto de Kronecker.

Demonstração: Por indução em $n$.

1) Para $n=1$ considere $\mathbf{A}$ uma matriz $A A_{2}$ dada por

$$
\mathbf{A}=\left[\begin{array}{cc}
z_{1} & z_{2} \\
-z_{2} & -z_{1}
\end{array}\right]
$$

Pode-se escrever A da seguinte forma:

$$
\begin{gathered}
\mathbf{A}=z_{1}\left[\begin{array}{cc}
1 & 0 \\
0 & -1
\end{array}\right]+z_{2}\left[\begin{array}{cc}
0 & 1 \\
-1 & 0
\end{array}\right] \\
=\frac{1}{2} z_{1}\left[\begin{array}{cc}
1+1 & 1-1 \\
-1+1 & -1-1
\end{array}\right]+\frac{1}{2} z_{2}\left[\begin{array}{cc}
1-1 & 1+1 \\
-1-1 & -1+1
\end{array}\right] .
\end{gathered}
$$

Logo, tem-se

$$
\mathbf{A}=\beta_{0}(\mathbf{A}) \mathbf{B}_{0}+\beta_{1}(\mathbf{A}) \mathbf{B}_{1}
$$

em que

$$
\begin{aligned}
& \beta_{0}(\mathbf{A})=\frac{1}{2}\left[z_{1}+z_{2}\right], \quad \beta_{1}(\mathbf{A})=\frac{1}{2}\left[z_{1}-z_{2}\right], \\
& \mathbf{B}_{0}=\left[\begin{array}{cc}
1 & 1 \\
-1 & -1
\end{array}\right] \quad \text { e } \quad \mathbf{B}_{1}=\left[\begin{array}{cc}
1 & -1 \\
1 & -1
\end{array}\right] .
\end{aligned}
$$

Observe que $\mathbf{B}_{0}=\mathbf{b}_{0}^{\prime} \otimes \mathbf{b}_{0}^{\prime \prime T}=\left[\begin{array}{c}1 \\ -1\end{array}\right] \otimes\left[\begin{array}{ll}1 & 1\end{array}\right]$,

$$
\mathbf{B}_{1}=\mathbf{b}_{1}^{\prime} \otimes \mathbf{b}_{1}^{\prime \prime T}=\left[\begin{array}{l}
1 \\
1
\end{array}\right] \otimes\left[\begin{array}{ll}
1 & -1
\end{array}\right],
$$

$\beta_{0}(\mathbf{A})=\frac{1}{2}\left[\begin{array}{ll}z_{1} & z_{2}\end{array}\right] \cdot \mathbf{b}_{0}^{\prime \prime}$ e $\beta_{1}(\mathbf{A})=\frac{1}{2}\left[\begin{array}{ll}z_{1} & z_{2}\end{array}\right] \cdot \mathbf{b}_{1}^{\prime \prime}$.

Note adicionalmente que o conjunto $\mathcal{B}=\left\{\mathbf{B}_{0}, \mathbf{B}_{1}\right\}$ é composto apenas por matrizes de posto unitário.

2) Hipótese de Indução

Seja A uma matriz $A A_{2^{k}}$ e seja o conjunto $\mathcal{B}_{2^{k}}=$ $\left\{\mathbf{B}_{1}, \mathbf{B}_{2}, \ldots, \mathbf{B}_{2^{k}}\right\}$, em que $\mathbf{B}_{j}, j=1,2, \ldots, 2^{k}$, é uma matriz de posto unitário. Considere que

$$
\mathbf{A}=\sum_{j=1}^{2^{k}} \beta_{j}(\mathbf{A}) \mathbf{B}_{j}
$$

em que $\mathbf{B}_{j}=\mathbf{b}_{j}^{\prime} \otimes \mathbf{b}_{j}^{\prime \prime T}, \mathbf{b}_{j}^{\prime}=\left[b_{i}^{\prime}\right]_{2^{k} \times 1}, \mathbf{b}_{j}^{\prime \prime}=$ $\left[b_{i}^{\prime \prime}\right]_{2^{k} \times 1}, b_{i}^{\prime} \in\{-1,+1\}, b_{i}^{\prime \prime} \in\{-1,+1\} \beta_{j}(\mathbf{A})=$ $\frac{1}{2^{k}}\left[\begin{array}{llll}z_{1} & z_{2} & \ldots & z_{2^{k}}\end{array}\right] \cdot \mathbf{b}_{j}^{\prime \prime}$ e $\left[\begin{array}{llll}z_{1} & z_{2} & \ldots & z_{2^{k}}\end{array}\right]$ é a primeira linha da matriz $\mathbf{A}$.
Para $n=2^{k+1}$, seja $\mathbf{A}$ uma matriz $A A_{2^{k+1}}$. Pela Definição 1 ,

$$
\mathbf{A}=\left[\begin{array}{cc}
\mathbf{A}_{11} & \mathbf{A}_{12} \\
-\mathbf{A}_{12} & -\mathbf{A}_{11}
\end{array}\right]
$$

em que $\mathbf{A}_{11}$ é $A A_{2^{k}}$, com a primeira linha dada por $\left\{z_{1}, z_{2}, \ldots, z_{2^{k}}\right\}$ e $\mathbf{A}_{12}$ é $A A_{2^{k}}$, com a primeira linha dada por $\left\{z_{2^{k}+1}, z_{2^{k}+2}, \ldots, z_{2^{k+1}}\right\}$. Assim pode-se escrever

$$
\mathbf{A}=\left[\begin{array}{cc}
\mathbf{A}_{11} & \mathbf{0} \\
\mathbf{0} & -\mathbf{A}_{11}
\end{array}\right]+\left[\begin{array}{cc}
\mathbf{0} & \mathbf{A}_{12} \\
-\mathbf{A}_{12} & \mathbf{0}
\end{array}\right]
$$

e, pela hipótese de indução em (2) e pela Definição 1,

$$
\begin{aligned}
\mathbf{A} & =\sum_{j=1}^{2^{k}}\left(\beta_{j}\left(\mathbf{A}_{11}\right)\left[\begin{array}{cc}
\mathbf{B}_{j} & \mathbf{0} \\
\mathbf{0} & -\mathbf{B}_{j}
\end{array}\right]\right) \\
& +\sum_{j=1}^{2^{k}}\left(\beta_{j}\left(\mathbf{A}_{12}\right)\left[\begin{array}{cc}
\mathbf{0} & \mathbf{B}_{j} \\
-\mathbf{B}_{j} & \mathbf{0}
\end{array}\right]\right)
\end{aligned}
$$

$$
\begin{aligned}
& =\frac{1}{2} \sum_{j=1}^{2^{k}}\left(\beta_{j}\left(\mathbf{A}_{11}\right)+\beta_{j}\left(\mathbf{A}_{12}\right)\right)\left[\begin{array}{cc}
\mathbf{B}_{j} & \mathbf{B}_{j} \\
-\mathbf{B}_{j} & -\mathbf{B}_{j}
\end{array}\right] \\
& +\frac{1}{2} \sum_{j=1}^{2^{k}}\left(\beta_{j}\left(\mathbf{A}_{11}\right)-\beta_{j}\left(\mathbf{A}_{12}\right)\right)\left[\begin{array}{cc}
\mathbf{B}_{j} & -\mathbf{B}_{j} \\
\mathbf{B}_{j} & -\mathbf{B}_{j}
\end{array}\right]
\end{aligned}
$$

Considere $\beta_{j 0}(\mathbf{A}) \triangleq \frac{1}{2}\left[\beta_{j}\left(\mathbf{A}_{11}\right)+\beta_{j}\left(\mathbf{A}_{12}\right)\right]$

$$
\begin{aligned}
& =\frac{1}{2^{k+1}}\left[\left[\begin{array}{llll}
z_{1} & z_{2} & \ldots & z_{2^{k}}
\end{array}\right] \cdot \mathbf{b}_{j}^{\prime \prime}\right. \\
& \left.+\left[\begin{array}{llll}
z_{2^{k}+1} & z_{2^{k}+2} & \cdots & z_{2^{k+1}}
\end{array}\right] \cdot \mathbf{b}_{j}^{\prime \prime}\right] \\
& =\frac{1}{2^{k+1}}\left[\begin{array}{llll}
z_{1} & z_{2} & \ldots & z_{2^{k+1}}
\end{array}\right] \cdot\left[\begin{array}{ll}
\mathbf{b} j^{\prime \prime T} & \mathbf{b} j^{\prime \prime T}
\end{array}\right]^{T} \\
& \text { e } \beta_{j 1}(\mathbf{A}) \triangleq \frac{1}{2}\left[\beta_{j}\left(\mathbf{A}_{11}\right)-\beta_{j}\left(\mathbf{A}_{12}\right)\right] \\
& =\frac{1}{2^{k+1}}\left[\left[\begin{array}{cccc}
z_{1} & z_{2} & \ldots & z_{2^{k}}
\end{array}\right] \cdot \mathbf{b}_{j}^{\prime \prime}\right. \\
& \left.-\left[\begin{array}{llll}
z_{2^{k}+1} & z_{2^{k}+2} & \cdots & z_{2^{k+1}}
\end{array}\right] \cdot \mathbf{b}_{j}^{\prime \prime}\right] \\
& =\frac{1}{2^{k+1}}\left[\begin{array}{llll}
z_{1} & z_{2} & \ldots & z_{2^{k+1}}
\end{array}\right] \cdot\left[\begin{array}{ll}
\mathbf{b} j^{\prime \prime T} & -\mathbf{b} j^{\prime \prime T}
\end{array}\right]^{T} \text {. }
\end{aligned}
$$

Sejam

$$
\mathbf{B}_{j 0}=\left[\begin{array}{cc}
\mathbf{B}_{j} & \mathbf{B}_{j} \\
-\mathbf{B}_{j} & -\mathbf{B}_{j}
\end{array}\right] \text { e } \mathbf{B}_{j 1}=\left[\begin{array}{cc}
\mathbf{B}_{j} & -\mathbf{B}_{j} \\
\mathbf{B}_{j} & -\mathbf{B}_{j}
\end{array}\right] .
$$

Como $\mathbf{B}_{j}=\mathbf{b}_{j}^{\prime} \otimes \mathbf{b}_{j}^{\prime \prime T}$, pode-se escrever

$$
\mathbf{B}_{j 0}=\mathbf{b}_{j 0}^{\prime} \otimes \mathbf{b}_{j 0}^{\prime \prime T} \quad \text { e } \quad \mathbf{B}_{j 1}=\mathbf{b}_{j 1}^{\prime} \otimes \mathbf{b}_{j 1}^{\prime \prime T},
$$

em que

$$
\begin{aligned}
& \mathbf{b}_{j 0}^{\prime}=\left[\begin{array}{c}
\mathbf{b}_{j}^{\prime} \\
-\mathbf{b}_{j}^{\prime}
\end{array}\right], \quad \mathbf{b}_{j 0}^{\prime \prime}=\left[\begin{array}{c}
\mathbf{b}_{j}^{\prime \prime} \\
\mathbf{b}_{j}^{\prime \prime}
\end{array}\right], \\
& \mathbf{b}_{j 1}^{\prime}=\left[\begin{array}{c}
\mathbf{b}_{j}^{\prime} \\
\mathbf{b}_{j}^{\prime}
\end{array}\right] \quad \text { e } \quad \mathbf{b}_{j 1}^{\prime \prime}=\left[\begin{array}{c}
\mathbf{b}_{j}^{\prime \prime} \\
-\mathbf{b}_{j}^{\prime \prime}
\end{array}\right] .
\end{aligned}
$$

Assim, 


$$
\mathbf{A}=\sum_{r=1}^{2^{k+1}} \beta_{r}(\mathbf{A}) \mathbf{B}_{r},
$$

em que, dado $\mathbf{z}=\left[\begin{array}{llll}z_{1} & z_{2} & \ldots & z_{2^{k+1}}\end{array}\right]$,

$$
\begin{aligned}
\beta_{r}(\mathbf{A}) & =\left\{\begin{array}{lc}
\mathbf{z} \cdot \mathbf{b}_{r 0}^{\prime \prime}, & 1 \leq r \leq 2^{k}, \\
\mathbf{z} \cdot \mathbf{b}_{r 1}^{\prime \prime}, & 2^{k}+1 \leq r \leq 2^{k+1}
\end{array}\right. \\
\mathbf{B}_{r} & =\left\{\begin{array}{lc}
\mathbf{B}_{r 0}, & 1 \leq r \leq 2^{k}, \\
\mathbf{B}_{r 1}, & 2^{k}+1 \leq r \leq 2^{k+1}
\end{array}\right.
\end{aligned}
$$$$
\mathrm{e}
$$

Definição 2: Sejam $\mathcal{B}_{1}=\left\{\mathbf{B}_{11}, \mathbf{B}_{12}, \ldots, \mathbf{B}_{1 m}\right\}$ e $\mathcal{B}_{2}=$ $\left\{\mathbf{B}_{21}, \mathbf{B}_{22}, \ldots, \mathbf{B}_{2 n}\right\}$ dois conjuntos de matrizes. Define-se o Produto de Kronecker desses dois conjuntos como sendo

$$
\mathcal{B}_{1} \otimes \mathcal{B}_{2}=\cup_{i=1}^{m} \cup_{j=1}^{n}\left\{\mathbf{B}_{1 i} \otimes \mathbf{B}_{2 j}\right\} .
$$

Posta esta definição, pode-se estabelecer o seguinte procedimento para a geração do conjunto $\mathcal{B}_{2^{n}}$ das matrizes de posto unitário, utilizadas no Teorema 1 , na decomposição das matrizes $A A_{2^{n}}$ :

Teorema 2: Seja $\mathcal{B}_{2^{n}}$ um conjunto de matrizes de posto unitário para decompor uma matriz $A A_{2^{n}}$, conforme Teorema 1. Para $n \geq 2$ tem-se $\mathcal{B}_{2^{n}}=\mathcal{B}_{2} \otimes \mathcal{B}_{2^{n-1}}$.

Demonstração: Pela Expressão (1) as matrizes $A A_{2}$ podem ser decompostas utilizando-se o conjunto de matrizes de posto unitário

$$
\mathcal{B}_{2}=\left\{\mathbf{B}_{0}, \mathbf{B}_{1}\right\}
$$

em que $\mathbf{B}_{0}=\left[\begin{array}{cc}1 & 1 \\ -1 & -1\end{array}\right]$ e $\mathbf{B}_{1}=\left[\begin{array}{cc}1 & -1 \\ 1 & -1\end{array}\right]$

Seja $\mathbf{A}$ uma matriz $A A_{2^{n}}$. Pelo Teorema 1 pode-se escrever

$$
\mathbf{A}=\sum_{r=1}^{2^{n}} \beta_{r}(\mathbf{A}) \mathbf{B}_{r}
$$

em que $\mathbf{B}_{r} \in \mathcal{B}_{2^{n}}$. Mas, por (3), cada matriz $\mathbf{B}_{r}$ pode ser escrita de uma das seguintes formas:

ou

$$
\mathbf{B}_{r}=\left[\begin{array}{cc}
\mathbf{B}_{j} & \mathbf{B}_{j} \\
-\mathbf{B}_{j} & -\mathbf{B}_{j}
\end{array}\right]=\mathbf{B}_{0} \otimes \mathbf{B}_{j}
$$

$$
\mathbf{B}_{r}=\left[\begin{array}{ll}
\mathbf{B}_{j} & -\mathbf{B}_{j} \\
\mathbf{B}_{j} & -\mathbf{B}_{j}
\end{array}\right]=\mathbf{B}_{1} \otimes \mathbf{B}_{j},
$$

em que $\mathbf{B}_{j} \in \mathcal{B}_{2^{n-1}}$. Assim $\mathcal{B}_{2^{n}}=\mathcal{B}_{2} \otimes \mathcal{B}_{2^{n-1}}$.

\section{AplicaÇões em Processamento de Sinais}

$\mathrm{O}$ tipo de simetria encontrado nas matrizes antiautossimilares pode ser utilizada para a obtenção de algoritmos rápidos. A ideia geral consiste em, dado um produto $\mathbf{X}=\mathbf{M x}$, em que $\mathbf{M}$ é uma matriz $2^{n} \times 2^{n}$ e $\mathbf{x}$ um vetor $2^{n} \times 1$, expressar a matriz $\mathbf{M}$ como a soma de uma matriz antiautossimilar e eventuais fatores de ajuste. Como existe uma forma sistemática de decompor uma matriz antiautossimilar em matrizes de posto-1, é possível obter algoritmos rápidos para alguns casos especiais de matrizes M. Nesta seção, como exemplos, são mostrados algoritmos rápidos para a computação da multiplicação de dois quatérnios e de dois octônios, que consistem em números hipercomplexos que têm sido recentemente estudados no contexto de processamento de sinais [3], [4], [5], [6] e [7].

\section{A. Quatérnios}

Um quatérnio sobre o corpo $\mathbb{R}$ é um número da forma

$$
a=a_{1} \mathbf{e}_{1}+a_{2} \mathbf{e}_{2}+a_{3} \mathbf{e}_{3}+a_{4} \mathbf{e}_{4},
$$

em que $a_{j} \in \mathbb{R}, j=1,2,3,4, \mathbf{e}_{1}=1$ e $\mathbf{e}_{j}, j=2,3,4$, são unidades imaginárias com multiplicações definidas segundo a Tabela I.

TABELA I

TABELA DE MUlTiPliCAÇÃo DAS UNidAdes IMAGINÁRIAS DE UM QUATÉRNIO.

\begin{tabular}{c||c|c|c|c|}
\hline$\times$ & $e_{1}$ & $e_{2}$ & $e_{3}$ & $e_{4}$ \\
\hline \hline$e_{1}$ & $e_{1}$ & $e_{2}$ & $e_{3}$ & $e_{4}$ \\
\hline$e_{2}$ & $e_{2}$ & $-e_{1}$ & $e_{4}$ & $-e_{3}$ \\
\hline$e_{3}$ & $e_{3}$ & $-e_{4}$ & $-e_{1}$ & $e_{2}$ \\
\hline$e_{4}$ & $e_{4}$ & $e_{3}$ & $-e_{2}$ & $-e_{1}$ \\
\hline
\end{tabular}

Por essa definição, o produto $a b$ de dois quatérnios, em que

$$
\begin{gathered}
a=a_{1} \mathbf{e}_{1}+a_{2} \mathbf{e}_{2}+a_{3} \mathbf{e}_{3}+a_{4} \mathbf{e}_{4}, \\
b=b_{1} \mathbf{e}_{1}+b_{2} \mathbf{e}_{2}+b_{3} \mathbf{e}_{3}+b_{4} \mathbf{e}_{4},
\end{gathered}
$$

pode ser representado como o produto matricial

$$
\mathbf{Y}=\mathbf{Q}_{4} \mathbf{X}
$$

em que

$$
\begin{gathered}
\mathbf{Y}=\left[-y_{1}, y_{2}, y_{3}, y_{4}\right]^{T}, \\
\mathbf{X}=\left[a_{1}, a_{2}, a_{3}, a_{4}\right]^{T}
\end{gathered}
$$

e

$$
\mathbf{Q}_{4}=\left[\begin{array}{cc|cc}
-b_{1} & b_{2} & b_{3} & b_{4} \\
b_{2} & b_{1} & b_{4} & -b_{3} \\
-- & -- & -- & -- \\
b_{3} & -b_{4} & b_{1} & b_{2} \\
b_{4} & b_{3} & -b_{2} & b_{1}
\end{array}\right]
$$

$\mathbf{X}$ representando o quatérnio $a$ e $\mathbf{Q}_{4}$ representando o quatérnio $b$.

Observa-se que, através do método direto, o produto de dois quatérnios pode ser computado utilizando-se 16 multiplicações e 12 adições.

A estratégia para obter um algoritmo rápido para multiplicação de quatérnios consiste em expressar $\mathbf{Q}_{4}$ numa soma de uma matriz antiautossimilar e fatores de ajuste, da seguinte forma:

$$
\mathbf{Q}_{4}=\mathbf{M}_{4}+2 \mathbf{F}_{4},
$$

em que

$$
\mathbf{F}_{4}=b_{1} \mathbf{F}_{4}(4,4)+b_{2} \mathbf{F}_{4}(1,2)+b_{3} \mathbf{F}_{4}(3,1)+b_{4} \mathbf{F}_{4}(2,3),
$$

$\mathbf{F}_{4}(r, s)=\left[f_{i j}\right]$, com

$$
f_{i j}=\left\{\begin{array}{rr}
1, & \text { se } i=r \text { e } j=s, \quad r, s \in\{1,2, \ldots, 4\}, \\
0, & \text { caso contrário }
\end{array}\right.
$$

e $\mathbf{M}_{4}$ está definida no Exemplo 1.

Note que a matriz composta por fatores de ajuste pode ser escrita como uma soma de 4 matrizes de posto unitário. 
Como $\mathbf{M}_{4}$ é $A A_{4}$, utiliza-se o Teorema 2 para gerar as matrizes utilizadas na decomposição de posto-1. Verifica-se primeiramente que

$$
\mathcal{B}_{2}=\left\{\mathbf{B}_{0}, \mathbf{B}_{1}\right\}
$$

em que

$$
\mathbf{B}_{0}=\left[\begin{array}{cc}
1 & 1 \\
-1 & -1
\end{array}\right] \text { e } \mathbf{B}_{1}=\left[\begin{array}{cc}
1 & -1 \\
1 & -1
\end{array}\right]
$$

Assim, pelo Teorema 2,

$$
\mathcal{B}_{4}=\mathcal{B}_{2} \otimes \mathcal{B}_{2}=\left\{\mathbf{B}_{00}, \mathbf{B}_{01}, \mathbf{B}_{10}, \mathbf{B}_{11}\right\}
$$

em que

$$
\begin{aligned}
\mathbf{B}_{00}= & {\left[\begin{array}{cccc}
1 & 1 & 1 & 1 \\
-1 & -1 & -1 & -1 \\
-1 & -1 & -1 & -1 \\
1 & 1 & 1 & 1
\end{array}\right], } \\
\mathbf{B}_{01}= & {\left[\begin{array}{cccc}
1 & -1 & 1 & -1 \\
1 & -1 & 1 & -1 \\
-1 & 1 & -1 & 1 \\
-1 & 1 & -1 & 1
\end{array}\right], } \\
\mathbf{B}_{10}= & {\left[\begin{array}{cccc}
1 & 1 & -1 & -1 \\
-1 & -1 & 1 & 1 \\
1 & 1 & -1 & -1 \\
-1 & -1 & 1 & 1
\end{array}\right], } \\
\mathbf{B}_{11} & =\left[\begin{array}{cccc}
1 & -1 & -1 & 1 \\
1 & -1 & -1 & 1 \\
1 & -1 & -1 & 1 \\
1 & -1 & -1 & 1
\end{array}\right] .
\end{aligned}
$$

De modo geral, ainda pode-se escrever

$$
\begin{gathered}
\mathbf{B}_{i j}=\mathbf{B}_{i} \otimes \mathbf{B}_{j}=\mathbf{b}_{i j}^{\prime} \otimes \mathbf{b}_{i j}^{\prime \prime T}, \quad i, j, \in\{0,1\}, \\
\mathbf{b}_{00}^{\prime T}=\left[\begin{array}{llll}
1 & -1 & -1 & 1
\end{array}\right], \quad \mathbf{b}_{00}^{\prime \prime T}=\left[\begin{array}{llll}
1 & 1 & 1 & 1
\end{array}\right], \\
\mathbf{b}_{01}^{\prime T}=\left[\begin{array}{llll}
1 & 1 & -1 & -1
\end{array}\right], \quad \mathbf{b}_{01}^{\prime \prime T}=\left[\begin{array}{llll}
1 & -1 & 1 & -1
\end{array}\right], \\
\mathbf{b}_{10}^{\prime T}=\left[\begin{array}{llll}
1 & -1 & 1 & -1
\end{array}\right], \quad \mathbf{b}_{10}^{\prime \prime T}=\left[\begin{array}{llll}
1 & 1 & -1 & -1
\end{array}\right], \\
\mathbf{b}_{11}^{\prime T}=\left[\begin{array}{llll}
1 & 1 & 1 & 1
\end{array}\right], \quad \mathbf{b}_{11}^{\prime \prime T}=\left[\begin{array}{llll}
1 & -1 & -1 & 1
\end{array}\right] .
\end{gathered}
$$

Pela Expressão (7) pode-se escrever o produto de dois quatérnios como

$$
\mathbf{Y}=\mathbf{Q}_{4} \mathbf{X}=\mathbf{M}_{4} \mathbf{X}+2 \mathbf{F}_{4} \mathbf{X}
$$

em que $\mathbf{M}_{4}$ é $A A_{4}$ e, como se pode observar em (8), $\mathbf{F}_{4}$ é uma soma de matrizes de posto unitário, de modo que $\mathbf{F}_{4} \mathbf{X}$ consiste em 4 termos de ajuste, em que cada termo de ajuste contribui com uma multiplicação na execução do algoritmo.

Portanto, a complexidade multiplicativa do algoritmo proposto é de oito multiplicações: quatro delas se referem à expansão da matriz antiautossimilar em matrizes de posto-1 e as quatro restantes resultam dos fatores de ajuste necessários para expressar a matriz $\mathbf{Q}_{4}$ por meio de uma matriz antiautossimilar. $\mathrm{O}$ algoritmo proposto tem a mínima complexidade multiplicativa conhecida na literatura [8] e está próximo do limite mínimo teórico de 7 multiplicações descoberto por Fiduccia [9].

\section{B. Octônios}

De acordo com [10] um octônio sobre o corpo $\mathbb{R}$ é um número da forma

$a=a_{1} \mathbf{e}_{1}+a_{2} \mathbf{e}_{2}+a_{3} \mathbf{e}_{3}+a_{4} \mathbf{e}_{4}+a_{5} \mathbf{e}_{5}+a_{6} \mathbf{e}_{6}+a_{7} \mathbf{e}_{7}+a_{8} \mathbf{e}_{8}$,

em que $a_{j} \in \mathbb{R}, j=1,2, \ldots, 8, \mathbf{e}_{1}=1$ e $\mathbf{e}_{j}, j=2, \ldots, 8$, são unidades imaginárias com multiplicação definida segundo a Tabela II.

TABELA II

TABela de MultipliCAÇão DAS UnidAdes IMAGINÁRIAS DE UM OCTÔNIO.

\begin{tabular}{c||c|c|c|c|c|c|c|c}
\hline$\times$ & $e_{1}$ & $e_{2}$ & $e_{3}$ & $e_{4}$ & $e_{5}$ & $e_{6}$ & $e_{7}$ & $e_{8}$ \\
\hline \hline$e_{1}$ & $e_{1}$ & $e_{2}$ & $e_{3}$ & $e_{4}$ & $e_{5}$ & $e_{6}$ & $e_{7}$ & $e_{8}$ \\
\hline$e_{2}$ & $e_{2}$ & $-e_{1}$ & $e_{4}$ & $-e_{3}$ & $e_{6}$ & $-e_{5}$ & $-e_{8}$ & $e_{7}$ \\
\hline$e_{3}$ & $e_{3}$ & $-e_{4}$ & $-e_{1}$ & $e_{2}$ & $e_{7}$ & $e_{8}$ & $-e_{5}$ & $-e_{6}$ \\
\hline$e_{4}$ & $e_{4}$ & $e_{3}$ & $-e_{2}$ & $-e_{1}$ & $e_{8}$ & $-e_{7}$ & $e_{6}$ & $-e_{5}$ \\
\hline$e_{5}$ & $e_{5}$ & $-e_{6}$ & $-e_{7}$ & $-e_{8}$ & $-e_{1}$ & $e_{2}$ & $e_{3}$ & $e_{4}$ \\
\hline$e_{6}$ & $e_{6}$ & $e_{5}$ & $-e_{8}$ & $e_{7}$ & $-e_{2}$ & $-e_{1}$ & $-e_{4}$ & $e_{3}$ \\
\hline$e_{7}$ & $e_{7}$ & $e_{8}$ & $e_{5}$ & $-e_{6}$ & $-e_{3}$ & $e_{4}$ & $-e_{1}$ & $-e_{2}$ \\
\hline$e_{8}$ & $e_{8}$ & $-e_{7}$ & $e_{6}$ & $e_{5}$ & $-e_{4}$ & $-e_{3}$ & $e_{2}$ & $-e_{1}$ \\
\hline
\end{tabular}

Por essa definição o produto $a b$, de dois octônios, em que $a=a_{1} \mathbf{e}_{1}+a_{2} \mathbf{e}_{2}+a_{3} \mathbf{e}_{3}+a_{4} \mathbf{e}_{4}+a_{5} \mathbf{e}_{5}+a_{6} \mathbf{e}_{6}+a_{7} \mathbf{e}_{7}+a_{8} \mathbf{e}_{8}$ $b=b_{1} \mathbf{e}_{1}+b_{2} \mathbf{e}_{2}+b_{3} \mathbf{e}_{3}+b_{4} \mathbf{e}_{4}+b_{5} \mathbf{e}_{5}+b_{6} \mathbf{e}_{6}+b_{7} \mathbf{e}_{7}+b_{8} \mathbf{e}_{8}$ pode ser representado como o produto matricial

$$
\mathbf{Y}=\mathbf{O}_{8} \mathbf{X}
$$

em que

$$
\begin{aligned}
& \mathbf{Y}=\left[-y_{1}, y_{2}, y_{3}, y_{4}, y_{5}, y_{6}, y_{7}, y_{8}\right]^{T}, \\
& \mathbf{X}=\left[a_{1}, a_{2}, a_{3}, a_{4}, a_{5}, a_{6}, a_{7}, a_{8}\right]^{T},
\end{aligned}
$$

$\mathrm{e}$

$$
\mathbf{O}_{8}=\left[\begin{array}{cccccccc}
-b_{1} & b_{2} & b_{3} & b_{4} & b_{5} & b_{6} & b_{7} & b_{8} \\
b_{2} & b_{1} & b_{4} & -b_{3} & b_{6} & -b_{5} & -b_{8} & b_{7} \\
b_{3} & -b_{4} & b_{1} & b_{2} & b_{7} & b_{8} & -b_{5} & -b_{6} \\
b_{4} & b_{3} & -b_{2} & b_{1} & b_{8} & -b_{7} & b_{6} & -b_{5} \\
b_{5} & -b_{6} & -b_{7} & -b_{8} & b_{1} & b_{2} & b_{3} & b_{4} \\
b_{6} & b_{5} & -b_{8} & b_{7} & -b_{2} & b_{1} & -b_{4} & b_{3} \\
b_{7} & b_{8} & b_{5} & -b_{6} & -b_{3} & b_{4} & b_{1} & -b_{2} \\
b_{8} & -b_{7} & b_{6} & b_{5} & -b_{4} & -b_{3} & b_{2} & b_{1}
\end{array}\right],
$$

$\mathrm{X}$ representando o octônio $a$ e $\mathbf{O}_{8}$ representando o octônio $b$.

Observa-se que, por meio do método direto, o produto de dois octônios pode ser computado utilizando-se 64 multiplicações e 56 adições.

A estratégia para obter um algoritmo rápido para multiplicação de octônios é análoga à utilizada na multiplicação de quatérnios e consiste em expressar $\mathbf{O}_{8}$ numa soma de uma matriz antiautossimilar e uma matriz composta por fatores de ajuste, da seguinte forma:

$$
\mathbf{O}_{\mathbf{8}}=\mathbf{M}_{\mathbf{8}}+2 \mathbf{F}_{8},
$$

em que 


$$
\begin{aligned}
& \mathbf{F}_{8}=b_{2} \mathbf{F}_{8}(1,2)+b_{4} \mathbf{F}_{8}(2,3)+b_{6} \mathbf{F}_{8}(2,5)+b_{7} \mathbf{F}_{8}(2,8) \\
& +b_{3} \mathbf{F}_{8}(3,1)+b_{7} \mathbf{F}_{8}(3,5)+b_{8} \mathbf{F}_{8}(3,6)+b_{1} \mathbf{F}_{8}(4,4) \\
& -b_{7} \mathbf{F}_{8}(4,6)-b_{5} \mathbf{F}_{8}(4,8)+b_{5} \mathbf{F}_{8}(5,1)+b_{3} \mathbf{F}_{8}(5,7) \\
& +b_{4} \mathbf{F}_{8}(5,8)-b_{8} \mathbf{F}_{8}(6,3)+b_{1} \mathbf{F}_{8}(6,6)-b_{4} \mathbf{F}_{8}(6,7) \\
& -b_{6} \mathbf{F}_{8}(7,4)-b_{3} \mathbf{F}_{8}(7,5)+b_{1} \mathbf{F}_{8}(7,7)+b_{8} \mathbf{F}_{8}(8,1) \\
& +b_{6} \mathbf{F}_{8}(8,3)+b_{5} \mathbf{F}_{8}(8,4)
\end{aligned}
$$

o elemento $f_{i j}$ da matriz $\mathbf{F}_{8}(r, s)$ é definido como

$$
f_{i j}=\left\{\begin{array}{rr}
1, & \text { se } i=r \text { e } j=s, \quad r, s \in\{1,2, \ldots, 8\}, \\
0, & \text { caso contrário }
\end{array}\right.
$$

e $\mathbf{M}_{8}$ está definida no Exemplo 2. Note que a matriz composta por fatores de ajuste pode ser escrita como uma soma de 22 matrizes de posto unitário.

Como $\mathbf{M}_{8}$ é uma matriz $A A_{8}$, utiliza-se o Teorema 2 para gerar as matrizes utilizadas na decomposição de posto-1. Assim,

$$
\begin{gathered}
\mathcal{B}_{8}=\mathcal{B}_{2} \otimes \mathcal{B}_{4}=\mathcal{B}_{2} \otimes \mathcal{B}_{2} \otimes \mathcal{B}_{2} \\
=\left\{\mathbf{B}_{000}, \mathbf{B}_{001}, \mathbf{B}_{010}, \mathbf{B}_{011}, \mathbf{B}_{100}, \mathbf{B}_{101}, \mathbf{B}_{110}, \mathbf{B}_{111}\right\},
\end{gathered}
$$

em que

$$
\begin{aligned}
& \mathbf{B}_{i j k}=\mathbf{B}_{i} \otimes \mathbf{B}_{j} \otimes \mathbf{B}_{k}=\mathbf{b}_{i j k}^{\prime} \otimes \mathbf{b}_{i j k}^{\prime \prime T}, \quad i, j, k \in\{0,1\}, \\
& \mathbf{B}_{0}=\left[\begin{array}{cc}
1 & 1 \\
-1 & -1
\end{array}\right] \quad, \quad \mathbf{B}_{1}=\left[\begin{array}{cc}
1 & -1 \\
1 & -1
\end{array}\right] \\
& \mathbf{b}_{000}^{\prime T}=\left[\begin{array}{llllllll}
1 & -1 & -1 & 1 & -1 & 1 & 1 & -1
\end{array}\right], \\
& \mathbf{b}_{000}^{\prime \prime T}=\left[\begin{array}{llllllll}
1 & 1 & 1 & 1 & 1 & 1 & 1 & 1
\end{array}\right], \\
& \mathbf{b}_{001}^{\prime T}=\left[\begin{array}{llllllll}
1 & 1 & -1 & -1 & -1 & -1 & 1 & 1
\end{array}\right], \\
& \mathbf{b}_{001}^{\prime \prime T}=\left[\begin{array}{llllllll}
1 & -1 & 1 & -1 & 1 & -1 & 1 & -1
\end{array}\right], \\
& \mathbf{b}_{010}^{\prime T}=\left[\begin{array}{llllllll}
1 & -1 & 1 & -1 & -1 & 1 & -1 & -1
\end{array}\right], \\
& \mathbf{b}_{010}^{\prime \prime T}=\left[\begin{array}{llllllll}
1 & 1 & -1 & -1 & 1 & 1 & -1 & -1
\end{array}\right], \\
& \mathbf{b}_{011}^{\prime T}=\left[\begin{array}{llllllll}
1 & 1 & 1 & 1 & -1 & -1 & -1 & -1
\end{array}\right], \\
& \mathbf{b}_{011}^{\prime \prime T}=\left[\begin{array}{llllllll}
1 & -1 & -1 & 1 & 1 & -1 & -1 & 1
\end{array}\right], \\
& \mathbf{b}_{100}^{\prime T}=\left[\begin{array}{llllllll}
1 & -1 & -1 & 1 & 1 & -1 & -1 & 1
\end{array}\right], \\
& \mathbf{b}_{100}^{\prime \prime T}=\left[\begin{array}{llllllll}
1 & 1 & 1 & 1 & -1 & -1 & -1 & -1
\end{array}\right] \text {, } \\
& \mathbf{b}_{101}^{\prime T}=\left[\begin{array}{llllllll}
1 & 1 & -1 & -1 & 1 & 1 & -1 & -1
\end{array}\right], \\
& \mathbf{b}_{101}^{\prime \prime T}=\left[\begin{array}{llllllll}
1 & -1 & 1 & -1 & -1 & 1 & -1 & 1
\end{array}\right] \text {, } \\
& \mathbf{b}_{110}^{\prime T}=\left[\begin{array}{llllllll}
1 & -1 & 1 & -1 & 1 & -1 & 1 & -1
\end{array}\right] \text {, } \\
& \mathbf{b}_{110}^{\prime \prime T}=\left[\begin{array}{llllllll}
1 & 1 & -1 & -1 & -1 & -1 & 1 & 1
\end{array}\right] \text {, } \\
& \mathbf{b}_{111}^{\prime T}=\left[\begin{array}{llllllll}
1 & -1 & -1 & 1 & 1 & -1 & 1 & -1
\end{array}\right], \\
& \mathbf{b}_{111}^{\prime \prime T}=\left[\begin{array}{llllllll}
1 & 1 & -1 & -1 & -1 & -1 & 1 & 1
\end{array}\right] \text {. }
\end{aligned}
$$

Pela Expressão (10) pode-se escrever o produto de dois octônios como

$$
\mathbf{Y}=\mathbf{O}_{8} \mathbf{X}=\mathbf{M}_{8} \mathbf{X}+2 \mathbf{F}_{8} \mathbf{X}
$$

em que $\mathbf{M}_{8}$ é $A A_{8}$ e, como se pode observar em (11), $\mathbf{F}_{8}$ é uma soma de matrizes de posto unitário, de modo que $\mathbf{F}_{8} \mathbf{X}$ consiste em 22 termos de ajuste, em que cada termo de ajuste contribui com uma multiplicação na execução do algoritmo.

Portanto, a complexidade multiplicativa do algoritmo proposto é de 30 multiplicações reais: oito delas se referem a expansão da matriz antiautossimilar em matrizes de posto1 e as vinte e duas restantes resultam dos fatores de ajuste necessários para expressar a matriz $\mathbf{O}_{8}$ por meio de uma matriz antiautossimilar. $\mathrm{O}$ algoritmo obtido possui a mínima complexidade multiplicativa observada na literatura [11].

\section{CONClusões}

Neste trabalho, foi introduzida uma nova forma de simetria matricial, através do conceito de matrizes antiautossimilares, Além disso, foi apresentado um procedimento sistemático para decompor uma matriz antiautossimilar em matrizes de posto unitário, no intuito de construir algoritmos rápidos em processamento digital de sinais. Como ilustração da técnica, foram propostos algoritmos rápidos para multiplicação de dois quatérnios e de dois octônios. A complexidade multiplicativa de ambos algoritmos obtidos é a mínima conhecida na literatura, mostrando a utilidade dos conceitos introduzidos.

\section{Agradecimentos}

O presente trabalho foi realizado com apoio da Coordenação de Aperfeiçoamento de Pessoal de Nível Superior - Brasil (CAPES) - Código de Financiamento 001 e do Conselho Nacional de Desenvolvimento Científico e Tecnológico (CNPq).

\section{REFERÊNCIAS}

[1] J. Lafon, Optimum computation of p bilinear forms, Linear Algebra and its Applications, v. 10 , n. 3 pp. 225-240, 1975.

[2] G. Jerônimo da Silva Jr. and R. M. Campello de Souza, Constructing Fast Algorithms by Expanding a Set of Matrices into Rank-1 Matrices, Circuits, Systems and Signal Processing, 39, 1630-1648, 2020.

[3] D. Alfsmann; H. G. Göckler, S. J. Sangwine, and T. A. Ell, Hypercomplex Algebras in Digital Signal Processing: Benefits and Drawbacks (Tutorial), Proc. EURASIP 15th European Signal Processing Conf EUSIPCO, Poznań, Poland, pp. 1322-1326, 2007.

[4] T.A. Ell, N. Le Bihan, S.J. Sangwine, Quaternion Fourier Transforms for Signal and Image Processing (Wiley, New York, 2014).

[5] F. Ortolani, D. Comminiello, M. Scarpiniti, and A. Uncini. Frequency domain adaptive filtering in hypercomplex systems. In 25th Italian Workshop on Neural Networks, 2015.

[6] Q. Yin, J. Wang, X. Luo, J. Zhai, S. K. Jha and Y. Shi, Quaternion Convolutional Neural Network for Color Image Classification and Forensics, in IEEE Access, vol. 7, pp. 20293-20301, 2019.

[7] F. Ortolani and A. Uncini, Quaternion digital signal processing: A hypercomplex approach to information processing, 2016 International Siberian Conference on Control and Communications (SIBCON), 2016.

[8] H. B. A. Barbosa, L. B. da Silva, G. Jerônimo da Silva Jr. e R. M. Campello de Souza, Um Algoritmo Rápido para a Multiplicação de Quatérnios, XXXVIII Simpósio Brasileiro de Telecomunicações e Processamento de Sinais, SBrT 2020, 22-25 de novembro de 2020, Florianópolis, SC.

[9] C. Fiduccia and Y. Zalcstein, Algebras Having Linear Multiplicative Complexities, Journal of the ACM (JACM), v. 24, pp. 311-331, April 1977.

[10] A. Cariow and G. Cariowa, Algorithm for multiplying two octonions, Radioelectronics and Communications Systems, v. 55, pp. 464-473, $\mathrm{n}^{\mathrm{O}}$ $10,2012$.

[11] H. B. A. Barbosa, L. B. da Silva, G. Jerônimo da Silva Jr. e R. M. Campello de Souza, Um Algoritmo Rápido para a Multiplicação de Octônios, XXXVIII Simpósio Brasileiro de Telecomunicações e Processamento de Sinais, SBrT 2020, 22-25 de novembro de 2020, Florianópolis, SC. 